\title{
Recommendations for prevention and control of new coronavirus infection in children with rheumatic diseases
}

\author{
Zeng Ping*, Wu Ping\#, Tang Hongxia ${ }^{\#}$, Yin Wei* and Zeng Huasong* \\ Paediatric Allergy, Immunology and Rheumatology Branch, Asia Pacific Association of Medicine and Bio-Immunology (PAIRB-APAMBI), China \\ ${ }^{\#}$ Co first Author, Contributed equally \\ *Correspondence author, Contributed equally
}

Experts (in order of initial strokes): the First People's Hospital of Guangzhou (Yu Li); Guangzhou Women and Children's Medical Center (Zeng Huasong, Zeng Ping, Li Feng); the First Affiliated Hospital of Guangxi Medical University (Nong Guangmin, Jiang Min); Guangdong People's Hospital (Lu Yiyun); Guangdong Second Provincial General Hospital (Zheng Shaoling); Shanghai Children's Medical Center, Shanghai Jiaotong University (Zhou Wei); Shanghai Children's Hospital, Shanghai Jiaotong University (Huang Wenyan, Hao Sheng); Qilu Children's Hospital of Shandong University (Zhang Hongxia); Dalian Municipal Women's and Children's Medical Center (Liu Yan); Tianjin Children's Hospital (Hu Jian); Urumqi Children's Hospital (Zhao Dongmei); Peking Union Hospital of Chinese Academy of Medical Sciences (Guan Kai); the First Affiliated Hospital of Sun Yat Sen University (Jiang Xiaoyun); the Second Affiliated Hospital of Sun Yat Sen University (Tan Weiping); the Third Affiliated Hospital of Sun Yat Sen University (Mu Yikun, Zhang Pingping); Zhongshan Boai Hospital Affiliated Southern Medical University (Li Xiaolin); Shengjing Hospital, China Medical University (Wu Yubin, Cai Xuxu, Du Yue); Children's Medical Center of Second Xiangya Hospital, Central South University (Yi Zhuwen, Wu Xiaochuan, Dang Xiqiang); the First People's Hospital of Yunnan Province (Wang Yajun); the Third Hospital of Peking University (Zhou Wei); Ningbo Women's and Children's Hospital (Di Yazhen); Children's Hospital of Beijing Capital Institute of Pediatrics (Chen Yuzhi); General Hospital of Ningxia Medical University (Lu Biao, Liang Lijun); Tongji Hospital, Tongji Medical College of Huazhong University of Science and Technology (Hu Xiufen, Qiu Liru); Wuhan Children's Hospital, Tongji Medical College of Huazhong University of Science and Technology (Yin Wei, Tang Hongxia); Anhui Children's Hospital (Chen Yuqing); the Second Affiliated Hospital of Shantou University Medical College (Lin Guangyu); X'an Third Hospital (Zhou Nan); Children's Hospital of X'an Jiaotong University (Li Xiaoqing); Children's Hospital of Jiangxi Province (Zou Zheng); First Hospital of Jilin University (Yang Sirui); Chengdu Women's and Children's Central Hospital (Zhang Wei); Children's Hospital of Suzhou University (Li Xiaozhong); Renmin Hospital of Wuhan University (Li Tao), Second Hospital of Hebei Medical University (Rong Zanhua); Children's Hospital of Hebei Province (Zhang Dongfeng); First Affiliated Hospital of Zhengzhou University (Peng Shao); Children's Hospital Affiliate to Zhengzhou University, Henan Children's Hospital , Zheng Zhou Children's Hospital(Liu,Cuihua); Children's Hospital of Kunming Medical University (Zhao Bo); Children's Hospital of Chongqing Medical University (Yang Xiqiang, Tang Xuemei); South Hospital of Southern Medical University (Sun Liangzhong); Zhujiang Hospital of Southern Medical University (Chen Jianfeng); Pediatrics Hospital of Fudan University (Xu Hong); the Second Affiliated Hospital of Harbin Medical University (Ren Lihong); Boston Children's Hospital of Harvard University (Li Peiyuan); Guiyang Children's Hospital (Shao Xiaoshan, Jiang Xinhui, Li Yuhong); Children's Hospital of Nanjing Medical University (Yu Haiguo); Prince of Wales Hospital of Chinese University of Hong Kong (Gary Huang); Children's Hospital of Medical Colleage, Zhejiang University (Lu Meiping, Xu Xuefeng); Shenzhen Children's Hospital (Li Yongbai); Hainan Women's and Children's Medical Center (Xu Zhiquan); the First Affiliated Hospital of Xiamen University (Xiao Jihong); The Second Affiliated Hospital \& Yuying Children's Hospital of Wenzhou Medical University (Zheng Wenjie); Fujian Provincial Hospital (Wang Tao); Hubei Maternal and Children Health Hospital (Wu Xiaolin); Hunan Children's Hospital (Li Zhihui); Hunan People's Hospital, Hunan Provincial People's Hospital, the First Affiliated Hospital of Hunan Normal University (Yang Manqiong); The First Affiliated Hospital of Xinxiang Medical College (Yang Dasheng); the First Affiliated Hospital of Jinan University (Yang Fang), Kiang Wu Hospital, Macau (Lee Cheng, Chen Yan, Chen Rongrong).

\begin{abstract}
During the epidemic period of sars-cov-2, 73 children's rheumatism immunology and clinical immunology experts from mainland China, Hong Kong, Macao and the United States were organized by the Paediatric Allergy, Immunology and Rheumatology Branch, Asia Pacific Association of Medicine and Bio-Immunology (PAIRB-APAMBI) (http://apambi.com/) The expert suggestion on novel coronavirus pneumonia management during childhood epidemic was carried out through Internet conferences. The clinical characteristics and differential diagnosis of sars-cov-2 infection in children with rheumatic diseases, the prevention of sars-cov-2 infection in children with rheumatic diseases, the management of specialized wards, the management of observation areas, the management of children and caregivers, During the epidemic period, it is necessary to go back and forth to the special hospital for the management and guidance of children with special treatment, management measures of related articles, treatment of suspected infection of sars-cov-2 in children with rheumatic diseases, treatment of confirmed infection of sars-cov-2 in children with rheumatic diseases, suggestions on special medicine, strengthening the management of chronic diseases and diversified diagnosis and treatment services, and establishing the management of chronic diseases during the epidemic period of sars-cov-2 System, online medical treatment and Internet of things system express special medicine, when the disease is aggravated, the guidance of medical treatment. This expert's suggestion has reference significance for the management of children with rheumatic diseases during the global epidemic of sars-cov- 2 .
\end{abstract}

${ }^{*}$ Correspondence to: Huasong Zeng, MD, PhD, Paediatric Allergy, Immunology and Rheumatology Branch, Asia Pacific Association of Medicine and Bio-Immunology (PAIRB-APAMBI), China, E-mail: huasongxuqing@163.com

Key words: SARS coronavirus 2, pneumonia, children, rheumatic diseases, management, prevention

Received: March 18, 2020; Accepted: March 24, 2020; Published: March 27, 2020 


\section{Review}

Rheumatic diseases in children are a group of common chronic diseases, which include diffuse connective tissue disease (Systemic lupus erythematosus, Juvenile dermatomyositis, etc.), juvenile chronic joint diseases (Juvenile idiopathic arthritis), systemic vasculitis and auto-inflammatory disease. Generally, these children often have an abnormal immune response to damage their own body and tissues. In order to control the damage of the immune system response to their bodies, these children should be treated with widely used glucocorticoids, immunosuppressive agents and biological agents. However, when the immune and inflammatory response has been suppressed, the capability of immune system to resistant pathogens will be significantly reduced. Some clinical study findings have revealed that the infants with idiopathic arthritis have a long-term usage of biological reagents Analakin, Tocilizumab, Etanercept, Infliximab, Adalimumab, Rituximab), immunosuppressive reagents (Methotrexate, Cyclosporine) and glucocorticoids. They may have a significantly increased risk of infection. Particularly, the younger of infants with biologic therapy, the more lymphocytes decrease, and the higher risk of infection when the child has a history of severe infection [1-4]. A variety of opportunistic infections include the infections from mycobacterium tuberculosis, fungi, herpes zoster virus, and so on $[5,6]$. The new coronavirus that caused the outbreak of viral pneumonia at Wuhan in December 2019. The International Virus Classification Commission has defined a name for the new coronavirus to be SARS-CoV-2, and the pneumonia caused by SARS-CoV-2 has been named COVID-19 [7]. So, the new coronavirus (2019-novel coronavirus, 2019-nCoV), which was the first time appeared in Wuhan, is a highly pathogenic human coronaviruses (HCoVs) [8]. HCoV infection is a zoonotic disease and poses a major threat to public health. However, we have no idea about the high invasiveness of this new coronavirus $[9,10]$. Coronaviruses (CoVs) are a large class of single positive strand RNA viruses, which belongs to the Coronaviridae family and the Nidovirus order. CoVs can be classified into $\mathrm{CoV} \alpha, \beta, \gamma$ and $\delta$ subtypes. These CoVs can infect a variety of hosts including birds, pigs, and humans. HCoVs are mainly CoVaand $\beta \mathrm{CoV}$. Under the electron microscope, the virus particles mostly appear to be a rough spherical or multi-faceted crystal, and the surface has a clublike spike, which is composed of spike proteins. Inside the virus particle there is a nucleocapsid enveloped virus genome, which contains about 26,000 to 32,000 bases pair nucleotide. Positive-stranded viral RNA can be used as messenger RNA (mRNA), including a cap structure at the 5 'end and multiple A-tail structures at the 3' end. Viral RNA mainly translates and replicates transcriptase. Replicase genes of all CoV species account for about two-thirds of the $5^{\prime}$-end RNA sequence. They consist of two overlapping ORFs, open reading frames (ORFs) 1a and ORF1b, which encode 16 non-structural proteins. The last one-third of the RNA sequence encodes the four classic structural proteins of the virus, which are Spike (also known as S protein), Envelope (also known as E protein), and Membrane (also known as M Protein) and nucleocapsid protein (also known as $\mathrm{N}$ protein). In addition, some auxiliary protein codes are also interspersed between structural protein coding regions, and different types of $\mathrm{CoV}$ auxiliary protein gene coding sites and numbers are reference standards for identifying different CoVs. At present, there is no study analyzing the incidence of sars-cov-2 infection in children with rheumatic diseases, but according to theoretical analysis, this type of children may be more susceptible to sars-cov-2, the clinical manifestations may be more severe, and the disease course is longer. Therefore, neonatal pneumonia may directly lead to higher mortality or related mortality than the general population. In the severe situation of epidemic prevention and control, it is particularly important to formulate strategies for the prevention and control of new coronavirus infections and clinical management in children with rheumatic diseases. At the end of 2002, the emergence of SARS-CoV infection in Guangdong, China, caused atypical infectious disease atypical pneumonia, which is characterized by symptoms such as fever, headache and cough. Subsequently, some patients can quickly develop severe respiratory failure and acute respiratory distress syndrome [11]. Due to the high human-to-human transmission capacity of SARS-CoV, the virus quickly spread to 29 countries, infected more than 8,000 cases, and had a mortality rate of approximately $10 \%$ [12]. Studies on genetic evolution of viral nucleotide sequences have determined that SARSCoV originated in bats [13]. In 2012, MERS-CoV appeared in Saudi Arabia, with clinical symptoms similar to SARS, but with a mortality rate of about 35\% [14]. The 2019-nCoV infection epidemic in Wuhan, China this time is also dominated by respiratory infection symptoms, but the mortality rate seems to be significantly lower than SARS-CoV and MERS-CoV infection, but human-to-human transmission has been confirmed, and the viral nucleotide sequence has been determined. But the true origin and intermediate host have yet to be determined $[15,16]$. This outbreak should attract great attention.

Since December 2019, the epidemic situation of new coronavirus infection has covered all parts of the country. Its pathogen is a singlestranded positive-strand RNA virus without segmentation, belonging to the genus Coronavirus $\beta$. The molecular mechanism of the interaction between S-protein and human ACE2 is used to infect human respiratory epithelial cells, resulting in a new type of coronavirus pneumonia $[11,15]$. The National Center for Disease Control and Prevention has announced that the disease will be included in the country's "Class B" infectious diseases and take "Class A" infectious disease prevention and control measures. Sars-cov-2 is mainly transmitted through respiratory droplets and can also be transmitted through contact. Epidemiological investigations suggest that the population is generally susceptible to sars-cov-2, the elderly and those with underlying diseases are more severely infected, and more and more children and infants are diagnosed. According to reports, the current youngest patient is only 1.5 months old [15]. Children with rheumatic diseases have basic diseases and immune dysfunction, need to take immunosuppressive drugs for a long time, and some children need to go to the hospital regularly for specialist treatment and other factors. They are susceptible to high-risk groups. Once infected, the condition is more difficult to control. It is necessary for these children to be highly vigilant and preventive against the new crown virus. In order to effectively prevent sars-cov-2 infection in children with this type of disease, and to control the primary disease after infection, we put forward the following prevention and control recommendations for children with rheumatic diseases, medication and prevention, for clinical reference.

\section{Clinical features and differential diagnosis of sars-cov-2 infection in children with rheumatic immune disease}

The infection of sars-cov-2 in children can be clinically asymptomatic, or fever, fatigue, dry cough, and a few children with upper respiratory symptoms such as nasal congestion, runny nose, and sore throat. Digestive symptoms such as abdominal discomfort, nausea, vomiting, abdominal pain, and diarrhoea may also occur. Laboratory tests showed that the total number of white blood cells in the peripheral blood was normal or decreased in the early stage of onset, the lymphocyte count decreased, and liver enzymes, muscle enzymes, and myoglobin increased in some patients. Most patients had elevated C-reactive protein and erythrocyte sedimentation rate and normal procalcitonin. In severe cases, D-dimer increases, and 
the number of peripheral blood lymphocytes decreases progressively. New coronavirus nucleic acids can be detected in pharyngeal swabs (nasopharyngeal swabs are recommended for children), sputum, lower respiratory tract secretions, and blood. Chest CT examination showed multiple small patchy shadows and interstitial changes at an early stage, which was evident with extrapulmonary bands. Further development of multiple ground glass and / or infiltrates in the lungs, severe cases of pulmonary consolidation, pleural effusion are rare $[7,13]$.

Children with rheumatic diseases may lack fever due to oral glucocorticoids, immunosuppressive agents, or biologics after infection with sars-cov-2, or they may only show symptoms such as cough, sore throat, nasal congestion, and runny nose, lacking typical symptoms. These symptoms need to be distinguished from respiratory infections caused by other pathogens (influenza virus, respiratory syncytial virus, adenovirus, mycoplasma infection, etc.), and winter and spring are the most frequent seasons for children with respiratory infections. In terms of laboratory tests, due to oral glucocorticoids, they do not show white blood cell decline or even increase. Inflammatory response indicators such as red blood cell sedimentation rate (ESR) and C-reactive protein (CRP) may not increase significantly or are normal. And due to long-term application of immunosuppressants can cause lymphocytopenia, and some patients with systemic lupus erythematosus during active disease may have clinical fever. When the blood system is involved, leukocytes and lymphocytes decline, rheumatic diseases can affect the lungs. And pulmonary lesions are usually manifested as interstitial pneumonia, but also as ground-glass changes. Due to the commonalities and similarities in clinical manifestations, laboratory tests, and imaging studies, it has brought difficulties to clinical diagnosis and differential diagnosis. Special attention should be paid to the differential diagnosis of children with rheumatic diseases combined with respiratory infections. It is necessary to analyze the clinical significance of laboratory examinations and influence imaging examinations based on the primary disease of the children, and to analyze epidemiological history, such as before the onset of disease. Travel history or residence history of Wuhan City and surrounding areas, or other case-reported communities within 14 days, contact history with new coronavirus infection (positive nucleic acid test) within 14 days before onset, contact with Wuhan and surrounding areas, or patients with fever or respiratory symptoms from a casereporting community, and clustered onset [14]. Highly suspected children should perform real-time fluorescent RT-PCR on respiratory specimens or blood specimens to detect novel coronavirus nucleic acids, sequence viral genes of respiratory specimens or blood specimens, and detect neocoronary pneumonia-specific antibodies (IgG or IgM) in blood to ensure that they are not misdiagnosed. No missed diagnosis, early diagnosis, early treatment, and early isolation [16-18].

\section{Prevention of children with rheumatic diseases}

\section{Management of specialist wards}

- Strengthen the training of all staff including doctors, nurses, and staff members on the prevention and control of sars-cov- 2 infection.

- Staff take temperature measurements twice a day before and after work and record them. If there is an abnormal body temperature, consult a doctor in time and arrange a rest at home.

- Strict implementation of hand hygiene. The timing of hand hygiene includes: (i) before and after direct contact with the child, or from a contaminated part of the same child's body to a clean area; (ii) before and after contact with the mucous membrane, damaged skin or wound of the child, contact with the child's blood, body fluids, secretions, excretion After dressing and wound dressing; (iii) before and after wearing the isolation gown, after removing gloves; (iv) before aseptic operation, contact with clean and sterile items; (v) after contact with the surrounding environment and items of children; (vi) before handling drugs or catering.

- A history of epidemiology should be reported in time and observed at home.

- Fever is a common symptom of rheumatism, and it is recommended to distinguish the disease into ordinary areas and observation areas. Patients with no epidemiological history and no fever were admitted to the general area. Patients with unclear epidemiological history and fever and coughing respiratory symptoms were admitted to the observation area.

$>$ General area management: The medical personnel who enter the general area for medical work are relatively fixed, and no longer enter the ward in the observation area for medical work. Manage in accordance with the daily management regulations of the ward, do a good job of disinfection of the area, do a good job of self-protection for medical staff, wear disposable surgical masks, hats, fast hand elimination, alcohol cotton balls, specialized stethoscopes, temperature guns, etc., and strictly perform hand hygiene. Ordinary area disinfection method: daily arrangement of cleaning staff to wear disposable surgical masks, hats, waterproof plastic gloves, prepare rapid hand disinfection solution, air disinfection (fixed air disinfection machine) 2 times / day, 30 minutes each time. $1000 \mathrm{mg} / \mathrm{L}$ chlorine-containing disinfectant is swabbed twice a day. Disinfection of the surface should be wiped with $1000 \mathrm{mg} / \mathrm{L}$ chlorine-containing disinfectant twice a day, and the disinfection time should be not less than 30 minutes.

$>$ Observation area management: The medical personnel who enter the observation area daily for medical work are relatively fixed and no longer enter the ward in the general area for medical work. Ward doctors, bed doctors, and nurses need to protect themselves before entering the observation ward. Wear disposable surgical masks, hats, fast hand wipes, alcohol cotton balls, specialized stethoscopes, and temperature guns to ensure good hand hygiene. Protective masks, face shields, or goggles should be worn for operations that are likely to produce aerosols such as sputum. Observation area disinfection method. Daily arrangement of cleaning staff to wear disposable surgical masks, hats, waterproof plastic gloves, disposable gowns, prepare rapid hand disinfection solution, air disinfection (fixed air disinfection machine) of the observation area 2 times / day, 30 minutes each time, floor disinfection with $2000 \mathrm{mg} / \mathrm{L}$ chlorine-containing disinfectant, 2 times / day, surface disinfection with $2000 \mathrm{mg} / \mathrm{L}$ chlorine-containing disinfectant, 2 times / day, disinfection time should not be less than 30 minutes.

\section{Management of children and caregivers}

- Actively carry out sars-cov-2 infection prevention knowledge education: provide hand hygiene, respiratory hygiene and cough etiquette guidance, improve the protection awareness of children and caregivers, strictly implement hand hygiene, and wash hands in accordance with the "seven-step hand-washing method". The timing of hand hygiene includes, before meals, after going to the toilet, after going home, after touching the garbage, after touching the animals. Try not to touch your mouth, nose and eyes before washing your hands. Remind children and caregivers to reduce going out and avoid crowded public places. Please try to stay at home if you are not 
in an emergency. When going out and taking public transportation, you must wear a mask. When you return home, you must promptly dispose of the mask, change your clothes, and wash your hands.

- Close contact with patients with new coronavirus infection and asymptomatic infection is the main way to cause infection in children. If the caregiver or family members have fever or respiratory symptoms, they need to closely monitor their temperature and clinical symptoms. Once abnormalities are found, they need to be designated hospital investigation. Carry outward isolation or home isolation observation according to the condition. The medical staff should be notified in advance before the specialist return. Medical staff will give guidance after inquiring about the history of epidemiological exposure.

- Ask the children and their families for epidemiological history and discomfort before going to the clinic. If the child is found to have fever and / or respiratory symptoms and a history of epidemiological exposure, he should be accompanied by a medical staff to the designated fever clinic. If the child's caregiver has fever and / or respiratory symptoms and has a history of epidemiological contact, immediately notify the caregiver to a designated adult hospital for a fever clinic.

- Every child should be accompanied by one caregiver as much as possible. Wear disposable surgical masks in public areas and keep a safe distance of more than 1 meter from others.

- During the epidemic, children who need to go to a specialist hospital for specialist treatment should not leave their place of residence as much as possible. Depending on the control of the primary disease, a specialist will formulate a plan based on the patient's condition and guide medication through WeChat and telephone to minimize the number of times patients travel to and from the hospital. No special circumstances, it is not recommended to receive children from other provinces and cities to cross-regional treatment.

- Insist on treatment, do not stop or stop the drug at will.

- Enhance resistance to physical strength: balanced nutrition, reasonable diet, and ensure adequate intake of high-quality protein, ensure adequate sleep, moderate exercise, and avoid excessive fatigue.

\section{Article management measures}

During the epidemic of sars-cov-2, in addition to doing a good job of cleaning and disinfecting medical equipment, contaminated articles, surfaces and floors, and disinfecting air in strict accordance with the "Technical Specifications for Disinfection of Medical Institutions" and "Code for Air Purification Management in Hospitals", it is necessary to strengthen Disinfection and isolation measures.

\section{Management of sars-cov-2 suspected infection in children with rheumatic diseases}

- Children with suspected sars-cov-2 infection: The local Center for Disease Control and Prevention should be notified immediately, and the child should be transferred to a designated institution with effective isolation and protection conditions. Suspected cases should be treated in a single room and isolated. For specific transfer plan, please refer to the work plan for transfer of pneumonitis cases of new coronavirus infection issued by the National Health and Health Commission (Trial) [19].
- Children who need medical observation: Children with rheumatic diseases who have close contact or suspicious exposure and medical staff need medical observation, the observation period is the last invalid protective contact with the case or suspicious exposure $14 \mathrm{~d}$. If the diagnosis of a suspected case is excluded, medical observation can be lifted [13].

> Medical observation method: The children with unstable primary disease are arranged to be isolated for medical observation in a single room in the hospital, and the specialists will continue to treat them.

$>$ Protective requirements for medical personnel: Specialized treatment for medical observation of children. Medical personnel should use at least secondary protection, wear work clothes, disposable hats, medical protective masks, protective clothing, goggles, shoe covers, double-layer latex gloves, and strictly implement hand hygiene.

$>$ During the observation period of suspected infection in children with rheumatic diseases, in order to avoid possible viral infections, immunosuppressants should be temporarily stopped temporarily. Children who are using large doses of glucocorticoids should be based on the primary disease and inflammation. Activity status, under the guidance of a specialist, gradually reduce the number of hormones or low-dose maintenance treatment.

$>$ Children whose primary disease is unstable or exacerbated should be detected as soon as possible, and blood purification treatment should be performed in time. The operation should be performed in accordance with the recommendations for prevention and control of new coronavirus infection in the Children's Blood Purification Center (Trial version 1) [20].

\section{Management of sars-cov-2 infection in children with rheumatic diseases}

Children diagnosed with sars-cov-2 infection should immediately notify the local Centers for Disease Control and Prevention and transfer the children to a designated institution with effective isolation and protection conditions and the ability to diagnose and treat children with rheumatic diseases. Multiple confirmed cases can be treated in the same ward. For children with immunosuppressive and immunocompromised functions, the damage caused by viral virulence may be heavier, longer, and the virus clearing time may be longer. In addition, the virus may activate the immune system, causing reactivation of autoimmune system diseases, and even inflammatory factor storms appear, making clinical treatment more difficult and complicated [21,22]. Therefore, on the basis of symptomatic treatment, we should actively prevent complications, treat underlying diseases, prevent secondary infections, and provide organ function support in a timely manner.

\section{Advice on specialist medication}

- Glucocorticoids: Glucocorticoids are the basic medication for controlling rheumatic diseases, but larger doses of glucocorticoids can delay the removal of coronavirus due to immunosuppressive effects. The current guidelines recommend that, as appropriate, according to the patient's breathing difficulty and chest imaging progress, as appropriate Short-term (3 to 5 days) use of glucocorticoids, the recommended dose does not exceed the equivalent of 1 to $2 \mathrm{mg} / \mathrm{kg}$ / day of methylprednisolone [23], due to long-term abnormalities in the immune status of children with rheumatic diseases, combined with infection after infection. The child's immune status and 
systemic conditions are determined by the child rheumatologist and the infection specialist based on the condition of the child. For children who have been taking hormones for a long time, if they are not suitable for continuing glucocorticoid therapy, they cannot be stopped suddenly. For physiological requirements, $15 \mathrm{mg} / \mathrm{m}^{2}$.

- Non-steroidal anti-inflammatory drugs: NSAIDs have different chemical structures, but they all exert their antipyretic, analgesic, and anti-inflammatory effects by inhibiting the synthesis of prostaglandins. Commonly used are ibuprofen, naproxen, loxoprofen sodium, celecoxib and the like. The main side effects are gastrointestinal adverse reactions, liver and kidney damage, avoid using two or more of these drugs at the same time. Patients with multiple organ dysfunction and gastrointestinal bleeding should be cautiously taken.

- Abiotic DMARDs: Also known as slow-acting antirheumatic drugs. The common feature of these drugs is that they have a slower onset of action. They can have anti-inflammatory and immune or immunosuppressive effects through different mechanisms, thereby improving joint swelling and stiffness, reducing systemic symptoms, and delaying joint and bone destruction. DMARDs include Chloroquine, Hydroxychloroquine, Sulfasalazine, Methotrexate, Azathioprine, Cyclophosphamide, Penicillamine, Gold preparations, Cyclosporine A, and Leflunomide. Patients with severe active infections should temporarily avoid the use of traditional DMARDs until the infection has subsided and antibiotic treatment has ended [24]. It is recommended that Hydroxychloroquine be used in children with rheumatism, and the conventional experience dosage is $5-7 \mathrm{mg} / \mathrm{kg}$ daily. Pay attention to monitoring blood routine and liver and kidney function. Based on adult experience, the sixth edition recommends Chloroquine phosphate for the treatment of new crown virus. The adult dose is $1 \mathrm{~g} /$ day $[25,26]$, which is 18 $20 \mathrm{mg} / \mathrm{kg}$ for children. In the short term, the accumulation exceeds the lethal amount [27]. The use of maintenance doses in children after the first dose is effective or short-term inhibition of virus expansion and cell-to-cell spread remains to be verified by further clinical studies.

- Biological DMARDs: At present, biological agents that can treat rheumatic diseases include tumor necrosis factor (TNF) - $\alpha$ antagonists, anti-CD20 monoclonal antibodies, interleukin (IL-1, IL-6) antagonists, and inhibitors of T cell co-stimulation signals. At present, tumor necrosis factor (TNF) - $\alpha$ antagonists commonly used in clinical practice are Etanercept, InfliXimab, Adalimumab, Golimumab. The anti-CD20 monoclonal antibody is Rituximab. Patients with severe active infections should temporarily avoid the use of biological DMARD and Tofacitinib until the infection has subsided and antibiotic treatment has ended [24].

- Intravenous immunoglobulin (IVIG): IVIG is one of the drugs for treating severe rheumatic diseases in children. Adult treatment experience in Wuhan is that IVIG has limited curative effect on sarscov-2 pneumonia. It can be considered for critically ill patients. The recommended dosage is $0.2 \mathrm{~g} /(\mathrm{kg} \cdot \mathrm{d})$, and the course of treatment is 3 to $5 \mathrm{~d}$ [23]. The commonly used dose in pediatrics is $400 \mathrm{mg} / \mathrm{kg}$ $\cdot d$, and the course of treatment is 3-5 days.

- Reasonable use of antibacterial drugs: Avoid blind or inappropriate use. Only in the case of secondary bacterial infection or secondary infection, under the premise of full microbiological examination, the corresponding antibacterial drug treatment is selected, and timely adjustments are made according to the efficacy and drug sensitivity test results.
- Blood purification treatment: For critically ill patients with high inflammatory response, it is necessary to identify as soon as possible. If possible, consider early use of extracorporeal blood purification techniques, including plasma exchange, blood adsorption, continuous renal replacement therapy [28].

- Psychotherapy: Patients often have anxiety and fear, and psychological counselling should be strengthened [29,30].

- Chinese traditional treatment: According to the condition, local climate characteristics and different physical conditions, dialectical treatment based on different ages [30].

\section{Strengthening management of chronic diseases and diversified diagnosis and treatment services}

- Establish a chronic disease management system to facilitate medical care to effectively monitor the situation of children and provide timely feedback, provide continuous care through platforms such as telephone return visits and WeChat group consultation to effectively and timely solve problems encountered by children at home. Children and caregivers unnecessarily travel to and from hospital during the cov-2 epidemic.

- Online consultation: Many domestic hospitals have opened online medical treatment platforms, plus public medical treatment platforms, online medical treatment has become very convenient. Instruct patients who have been diagnosed to use online medical care, and to go online for medical treatment under stable conditions. Follow-up, medicines can be sent to home to reduce unnecessary outings.

- Maintain the original treatment plan and purchase medicines nearby: If the condition is stable and you can't contact the consultation platform or doctor at present, you can purchase medicines from the nearest pharmacy according to the original treatment plan, and continue the treatment according to the original treatment plan. After the epidemic situation, you can go to the clinic.

- Hospital visits: If the condition worsens, you need to do laboratory tests and imaging examinations. Contact your doctor or specialist platform in time to see if you can see there. If you can't contact or can't receive treatment, find a nearby good rheumatology department. See a doctor. However, pay attention to wearing a mask, keep a certain distance from other patients, avoid cross infection, and cooperate with the hospital triage to inform the medical staff of the history of rheumatism and related contact history in detail.

\section{References}

1. Barut K, Adrovic A, Sahin S, Tarcin G, Tahaoglu G, et al. (2019) Prognosis, complications and treatment response in systemic juvenile idiopathic arthritis patients: A single-center experience. Int J Rheum Dis 22: 1661-1669. [Crossref]

2. Sengler C (2019) New therapy approaches, better outcomes? : Results from inception cohorts for patients with juvenile idiopathic arthritis. Z Rheumatol 78: 610-619. [Crossref]

3. Horneff G (2019) New drugs for treatment of juvenile idiopathic arthritis. Z Rheumatol 78: 587-598.

4. Klein A (2019) Biologics in the treatment of juvenile idiopathic arthritis: A comparison of mono- and combination therapy with synthetic DMARDs. Z Rheumatol 78: 599-609. [Crossref]

5. Brunelli J, Schmidt AR, Sallum AME, Goldenstein-Schainberg C, Bonfa E, et al (2016) High Rate of Serious Infection in Juvenile Idiopathic Arthritis Under Biologic Therapy in a Real-Life Setting [abstract]. Arthritis Rheumatol 68: suppl 10. [Crossref]

6. Giancane G, Swart J, Bovis F, Castagnola E, Groll A et al. (2016) Risk of Infections in Juvenile Idiopathic Arthritis Patients Treated with Biologic Agentsand/or Methotrexate: Results from Pharmachild Registry [abstract]. Arthritis Rheumatol 68: suppl 10. [Crossref] 
7. Gorbalenya AE, Baker SC, Baric RS, de Groot RJ, Drosten C, et al. (2020) Severe acute respiratory syndrome-related coronavirus: The species and its viruses - a statement of the Coronavirus Study Group. Biorxiv. 937862. [Crossref]

8. Jernigan DB (2020) CDC COVID- 9 Response Team; Update: Public Health Response to the Coronavirus Disease 2019 Outbreak - United States, February 24, 2020. Morb Mortal Wkly Rep. 69: 216-219. [Crossref]

9. Xia JG, Zhao JP, Cheng ZS, Hu Y, Duan J, et al. (2020) Non-invasive respiratory support for patients with novel coronavirus pneumonia: clinical efficacy and reduction in risk of infection transmission. Chin Med J (Engl) 761. [Crossref]

10. Chen W, Lan Y, Yuan X, Deng X, Li Y, et al. (2020) Detectable 2019-nCoV viral RNA in blood is a strong indicator for the further clinical severity. Emerg Microbes Infect 9: 469-473. [Crossref]

11. Office of the State Administration of Traditional Chinese Medicine, General Office of the National Health and Health Commission. Notice on Printing and Distributing Pneumonia Diagnosis and Treatment Plan for New Coronavirus Infection (Trial Version 4). 2020. Http://www.gov.cn/zhengce/zhengceku /2020/01/28/content_5472673.html [Crossref]

12. Tong ZD, Tang A, Li KF, Li P, Wang H, et al. (2020) Potential pre-symptomatic transmission of SARS-CoV-2, Zhejiang Province, China, 2020. Emerg Infect Dis 26: 198. [Crossref]

13. Jiang Yi, Runming J, Yuejie Z, et al. (2020) Expert consensus on diagnosis, treatment and prevention of children with new type of coronavirus infection (first edition). Chinese Journal of Practical Pediatrics 35: 81-85. [Crossref]

14. Office of the State Administration of Traditional Chinese Medicine, General Office of the National Health and Health Commission. Notice on Issuing a New Coronary Virus Pneumonia Diagnosis and Treatment Plan (Trial Version 6). 2020. http://www.nhc.gov. cn/xcs/zhengcwj/202002/8334a8326dd94d329df351d7da8aefc2.shtml [Crossref]

15. Zhu N, Zhang D, Wang W, Li X, Yang B, et al. (2020) A Novel Coronavirus from Patients with Pneumonia in China, 2019. N Engl J Med 382: 727-733. [Crossref]

16. Zuo MZ, Huang YG, Ma WH, Xue ZG, Zhang JQ, et al. (2020) Expert Recommendations for Tracheal Intubation in Critically ill Patients with Noval Coronavirus Disease 2019. Chin Med Sci J 3724. [Crossref]

17. Liu Z, Xiao X, Wei X, Li J, Yang J, et al. (2020) Composition and divergence of coronavirus spike proteins and host ACE2 receptors predict potential intermediate hosts of SARS-CoV-2. J Med Virol. 25726. [Crossref]

18. Zhu Y, Chen L, Ji H, Xi M, Fang Y, et al. (2020) The Risk and Prevention of Novel Coronavirus Pneumonia Infections Among Inpatients in Psychiatric Hospitals. Neurosci Bull 36: 299-302. [Crossref]
19. General Office of the National Health and Health Commission. Working plan for the transfer of pneumonitis cases with new coronavirus infection (trial). 2020. http: //www. gov.cn/zhengce/zhengceku/2020-01 / 29 / content_5472894.html [Crossref]

20. Prompetchara E, Ketloy C, Palaga T (2020) Immune responses in COVID-19 and potential vaccines: Lessons learned from SARS and MERS epidemic. Asian Pac J Allergy Immunol 38: 1-9. [Crossref]

21. Expert advice for the management of children with rheumatic diseases during the epidemic of the new coronavirus pneumonia. Chinese Journal of Practical Pediatrics. [Crossref]

22. Dongchi Z, Runming J, Zhisheng L, et al. (2020) Recommendations for the diagnosi and treatment of children with new type of coronavirus infection in Hubei Province (trial version). Chinese Journal of Contemporary Pediatrics 22: 96-99. [Crossref]

23. Singh JA, Furst DE, Bharat A, Curtis JR, Kavanaugh AF, et al. (2012) 2012 update of the 2008 American College of Rheumatology recommendations for the use of diseasemodifying antirheumatic drugs and biologic agents in the treatment of rheumatoid arthritis. Arthritis Care Res (Hoboken) 64: 625-639. [Crossref]

24. Guangdong Provincial Department of Science and Technology and Guangdong Provincial Health and Health Committee chloroquine phosphate multi-center collaborative group for the treatment of new coronavirus pneumonia (2020) Expert consensus on chloroquine phosphate for the treatment of new coronavirus pneumonia Chinese Journal of Tuberculosis and Respiratory Diseases 43: E019-E019. [Crossref]

25. Gao J, Tian Z, Yang X (2020) Breakthrough: Chloroquine Phosphate Has Shown Apparent Efficacy in Treatment of COVID-19 Associated Pneumonia in Clinical Studies. Biosci Trends 14: 72-73. [Crossref]

26. Schrezenmeier E, Dorner T (2020) Mechanisms of action of hydroxychloroquine and chloroquine: implications for rheumatology. Nat Rev Rheumatol 16: 155-166. [Crossref]

27. Liu XM, Wang DQ (2020) Consideration and suggestions on development of blood transfusion department under the epidemic situation of novel coronavirus pneumonia. Zhonghua Yi Xue Za Zhi 100: E013. [Crossref]

28. Xiao C (2020) A Novel Approach of Consultation on 2019 Novel Coronavirus (COVID-19)-Related Psychological and Mental Problems: Structured Letter Therapy. Psychiatry Investig 17: 175-176. [Crossref]

29. Park SC, Park YC (2020) Mental Health Care Measures in Response to the 2019 Nove Coronavirus Outbreak in Korea. Psychiatry Investig 17: 85-86. [Crossref]

30. Jia WP (2020) Strengthen comprehensive strategies to treat patients with mild novel coronavirus pneumonia. Zhonghua Nei Ke Za Zhi 59: E002. [Crossref]

Copyright: (C2020 Ping Z. This is an open-access article distributed under the terms of the Creative Commons Attribution License, which permits unrestricted use, distribution, and reproduction in any medium, provided the original author and source are credited. 